\title{
THE ONE STEP FORWARD LATERAL SPINAL X-RAY: MEASUREMENT OF SAGITTALAND SPINOPELVIC PARAMETERS IN A FUNCTIONAL POSITION
}

\author{
๑ Ümit Özgür GÜLER' 1 ๑ Emre ACAROĞLU² \\ ${ }^{1}$ Başkent University Adana Training and Research Hospital, Clinic of Orthopedics and Traumatology, Adana, Turkey \\ ${ }^{2}$ Ankara Spine Center, Clinic of Orthopedics and Traumatology, Ankara, Turkey
}

\begin{abstract}
Objective: Previous studies demonstrated an association between health related quality of life and sagittal balance for the adult spinal deformity (ASD) population, rendering an accurate evaluation of sagittal balance very important. Patients develop compensatory extension of hips, retroversion of pelvis and flexion of knees, identification and elimination of which may be useful. One step forward (OSF) is a lateral X-ray taken with the patient in the "starting to walk" position, taking the first step forward. To compare the sagittal balance and spinopelvic parameters between regular lateral and OSF X-rays in an ASD population and in those with increased pelvic retroversion [pelvic tilt (PT) >25].

Materials and Methods: Forty seven patients with ASD enrolled had their standing lateral X-rays in regular and OSF positions. OSF was defined as that with the patient taking one full step forward with the self-preferred side. Sagittal spinal and spinopelvic parameters were measured. Comparisons were made for the entire population and for patients with PT >25 degrees.

Results: Of 47 patients, 17 had PT >25 degrees. OSF did not create any effect in the general population but did so in PT >25 patients for spinopelvic angle, spinosacral angle and global tilt and for sacral slope, PT and pelvic incidence.

Conclusion: As evidenced by a decrease in the PT values, OSF eliminates the compensatory pelvic retroversion. It would be reasonable to accept the measurements in OSF as the more "functional" measurements. It is also probable that using OSF in surgical planning may decrease the possibility of imbalance.
\end{abstract}

Keywords: Sagittal balance, adult, radiography

\section{INTRODUCTION}

Adult spinal deformity is getting to be recognized as a real health and social problem as our population ages. The standard initial radiodiagnostic test for this population is the standing antero-posterior (AP) and lateral radiographs of the entire spine. Although the "normal" coronal plane of the spine is almost similar for every person, sagittal plane does not have a single normal alignment and nor is necessarily static during the life span. A better understanding the importance and impact of sagittal balance on functional outcomes and patient satisfaction as well as the results of treatment in later years have prompted the definition of several sagittal plane spinopelvic parameters to be taken on standing lateral whole spine radiographs from occiput to hip joints ${ }^{(11)}$. It is now well know that inappropriate evaluation of sagittal balance before the surgery may result in flatback, accelerated adjacent segment degeneration, pain and inferior outcomes in terms of health related quality of life (HRQL) instruments ${ }^{(1,2,11,15)}$. To achieve optimum results after surgery, maximum awareness of the potential problems of preoperative and postoperative sagittal balance appears to be essential. With regard to the global sagittal spinal alignment, C7 positioning is accepted for its stability over the sacrum in asymptomatic population. Of note, most people as well as patients with spinal deformity tend to acquire a positive sagittal balance (that is, the gravity line shifting forward) as a result of aging and/or spinal deformity. Several studies have shown that this change in balance is one of the most important parameters affecting the HRQL especially in people with adult spinal deformity ${ }^{(5,7,11,15)}$. It is also evident that every person affected by such a change in the sagittal spinal balance would recruit several compensatory mechanisms, the most frequent being the flexion of the pelvis by extending the hip joints followed by flexion of the knee joints. In radiographical evaluation of the sagittal balance, flexion of the knees is usually (supposedly) eliminated by the X-ray technicians whereas the flexion of the pelvis is not eliminated and can be evidenced by an increased pelvic tilt (PT) ${ }^{(10,11)}$. These standard $\mathrm{X}$-rays used to evaluate deformity and balance are static and 
can only show the position of the patient in a standing upright position at best. This compensated position however, may not necessarily be maintaned when the patient starts to walk, as one of the hips (the opposite side of the hip taking the step) has to be over-extended in due process ${ }^{(3,6,11,12)}$. Therefore, especially in patients who had been compensating for their positive sagittal balance by hip hyperextension (to the limit) or in those with pathologies of the hip (i.e. osteoarthritis) the actual walking position may be (has to be) that of more positive sagittal balance than reflected by standard standing $\mathrm{X}$-rays. This indeed is a common observation for those dealing with elderly; they can stand somewhat upright, but can not maintain this position while walking(5,6,8,10). We hypothesized that this walking position (reflecting the real sagittal alignment) may be mimicked by having the patient take a step forward at the time $X$-rays are taken. To the best of the author's knowledge, there is no study that has evaluated the effect of walking position on the segmental, regional, and global sagittal spinal alignment. The aim of the present study was to compare sagittal balance and parameters used commonly to determine sagittal balance between two different techniques of lateral whole spine radiographs [standard technique and one step forward (OSF) technique], in adult patients with spinal deformity.

\section{MATERIALS AND METHODS}

This study was based on a prospective spinal centre database of 47 adult spinal deformity patients (39 female, 8 male) who were scheduled to undergo full lenght AP and lateral spine radiographs for either surgical or conservative treatment. The inclusion criteria were: 1) Adult patients with deformity of the spinal column with or without spinal stenosis in which spinal deformity is defined as the presence of any of the following a) Coronal plane deformity $>25$ degrees, b) thoracic kyphosis (TK) $>60$ degrees, c) PT >25 degrees and d) Spinal vertical axis (SVA) $>50 \mathrm{~mm}$; 2) Patients who are willing to sign an informed consent to participate the study. On the other hand, patients who had previous servical, thoracal or lumbar surgeries (fusion and/or instrumentation) and patients who have documented severe hip and knee problems such as previous surgery or being scheduled for surgery were excluded from the study. Digital lateral whole spine radiographs were obtained from each patient in two different positions preoperatively. In the standard technique, patients stand in front of the X-ray sensor holding bars in front of them with the shoulders in 30 degrees of flexion. Legs are paralel in the position of patients own preference when standing. In the OSF technique, patient will take a step forward of up to $25 \mathrm{~cm}$ (ideally should be larger than the foot size), with the leg she/he prefers or the side of hip pain if any, in order to fix the hip joints and will stand in front of the sensor also holding the bars in front (Figure 1, 2) (3,4,9,11). SVA, TK, lumbar lordosis (LL), sacral slope (SS), PT, pelvic incidence (PI), spinopelvic angle (SPA), spinosacral angle (SSA), kyphosis tilt angle, spinal tilt angle (STA), T1 spinopelvic inclination (T1-SPI) and T9-SPI were measured for all patients in both positions using a specific digital X-ray analysis software (Surgimap, Beta 1.2.1.56, USA) ${ }^{(2,5,7,10,12)}$. Numerical variables were reported as mean + standard error of the mean, minimum to maximum range. Student's t-test with repeated measures and two tails was used for the statistical comparisons of standing versus OSF measurements in two different age groups of younger or older than 40 years. A p value of $<0.05$ was considered to be significant.

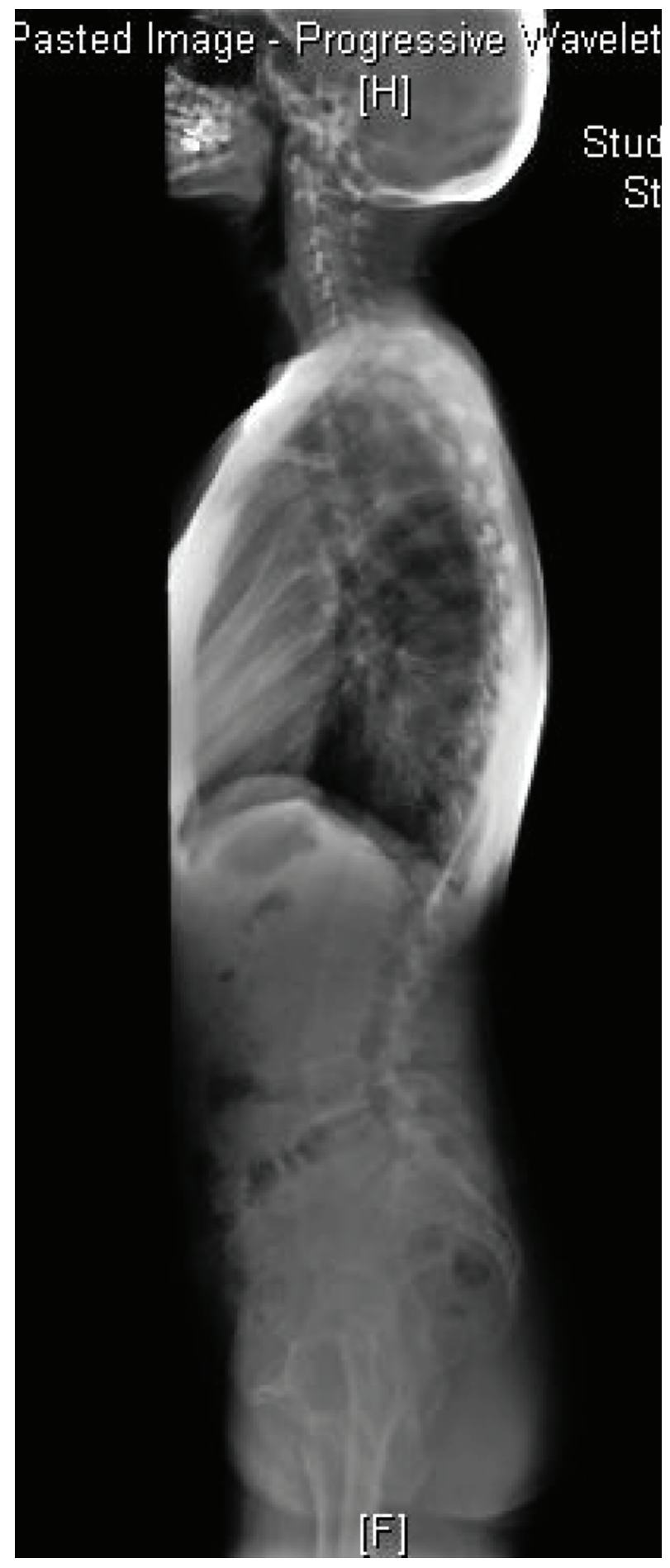

Figure 1. Whole spine lateral X-ray 


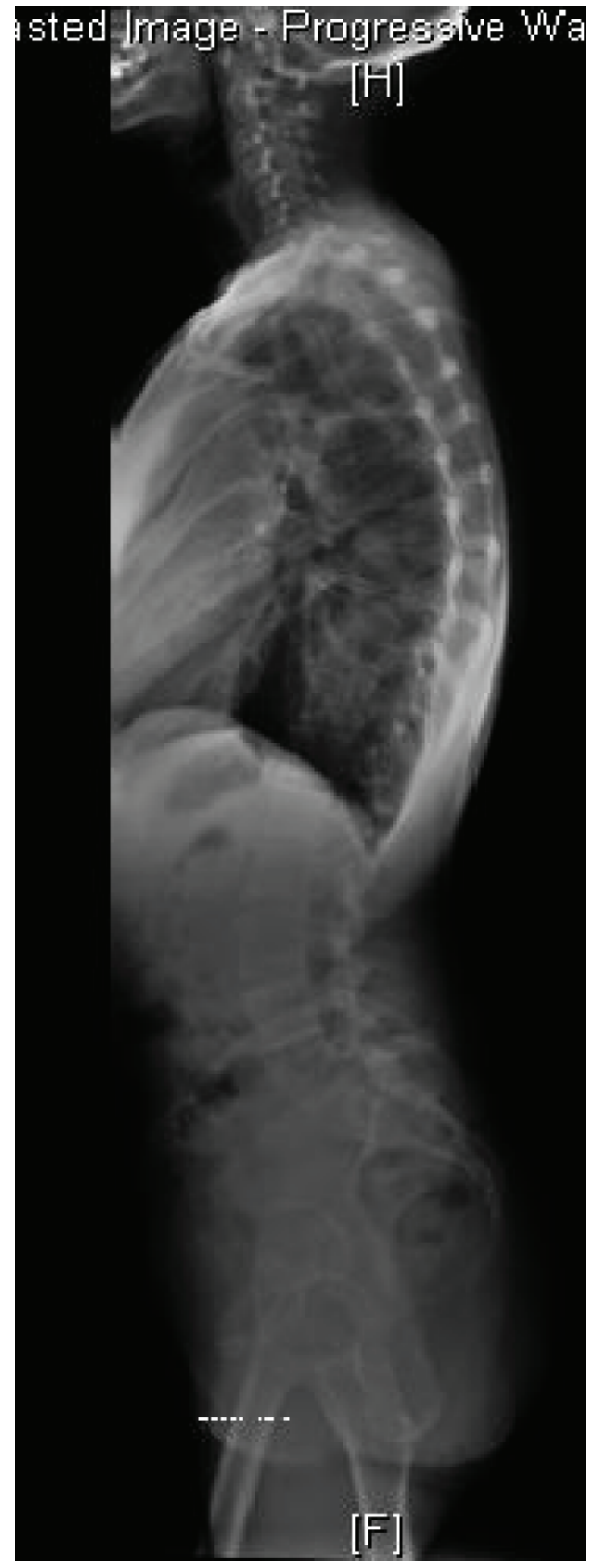

Figure 2. Whole spine one step forward lateral X-ray of same patient

\section{RESULTS}

The results are summarized in table 1 . In this study, we retrospectively evaluated 47 patients of both genders (8 male, 39 female). Ten patients were under 40 years old and 37 patients were over 40 years. Average age was 54.4. Evaluated standard standing lateral X-ray and OSF lateral X-ray sagittal balance and spinopelvic parameters did not show any statistically significant difference in whole study group (47 patients); but in subgroup of patients with increased PT (17 patients with PT $>25$ ) we found significant differences in SPA, SSA, global tilt (GT) and SS, PT and PI parameters. In increased PT subgroup, PT values mean 34.1 degrees with standard $X$-rays, showed a decrease to 18.5 degrees with OSF $X$-rays $(p<0.001)$ which was the most statistically significant difference in our study. SS values average increased from 26.8 to 34.2 degrees $(p<0.002$ ), while PI average decreasing from 61.2 to 53 degrees $(p<0.05)$. Also as seen in table 1 , decreased mean values of SPA, SSA and GT parameters with OSF lateral X-ray were statistically significant. However changes in SVA, TK, LL, STA mean values were found as insignificant.

\section{DISCUSSION}

This study aimed to analyze the differences in sagittal balance parametrs in two different positions for an adult deformity population ${ }^{(8,10,14)}$. Our hypothesis was that by having the patients take OSF during the sagittal plane X-rays, the hip extension/ pelvic rotation compensation of patients may be eliminated and results closer to the real life extent of the imbalance may be obtained(11). Our results appear to support our hypothesis in general. It is demonstrated that SVA, PT parameters do significantly worsen as the patients takes OSF, especially in the population older that 40 years of age. In line with our hypothesis, our result confirm that OSF lateral radiographs are effective in eliminating the compensation mechanisms used by the patient to stand upright thus giving a more realistic picture of the sagittal balance problem. These X-rays are closer to the clinical picture of the patient and may as well be closer to the end balance of the patient after surgery (given that surgery is not planned based on them). Having thus established the efficacy in reflecting the pre-treatment pathology more accurately than standard lateral X-rays, further studies focusing on the predictive value of the sagittal balance following surgery will be needed ${ }^{(4,9,13,14)}$. The major shortcoming of this study is the limited number of patients who had consented to have an additional X-ray for research purposes. On the other hand, as significant differences could be demonstrated, potential problems with sample size (i.e. limited statistical power) appear not to be particularly relevant. On the other hand, further studies comparing pre-treatment to post-treatment results may have to be done with larger sample sizes.

\section{CONCLUSION}

In this study; it has shown that lateral standing X-rays taken with the patients taking OSF demonstrate significantly higher levels of imbalance in adult deformity patients. The mechanism behind this is most probably the elimination of compensatory hip extension and pelvic flexion by that step. In this respect, OSF lateral X-rays may be closer to the actual clinical picture 
Table 1. Analysis of sagittal balance and spinopelvic parameter changes between standard lateral and one step forward lateral $\mathrm{X}$-rays in adult spinal deformity patients and in increased pelvic tilt subgroup

\begin{tabular}{lllllll}
\hline & Lateral & OSF lateral & p value & Lateral (PT > 25) & OSF (PT >25) & p value (PT > 25) \\
\hline SVA & $51.8(50.9)$ & $63.6(54.2)$ & 0.29 & $66.3(58.5)$ & $46.6(42.7)$ & 0.30 \\
\hline TK & $39.0(31.0)$ & $34.8(29.7)$ & 0.40 & $29.2(16.8)$ & $29.7(12.2)$ & 0.93 \\
\hline LL & $48.9(27.8)$ & $45(22.7)$ & 0.49 & $37.4(28.7)$ & $51.7(25.5)$ & 0.16 \\
\hline SPA & $25.8(16.4)$ & $26.6(17.1)$ & 0.74 & $40.2(13.2)$ & $20.9(14.3)$ & $\mathbf{0 . 0 0 3 ^ { * }}$ \\
\hline SSA & $59.9(14.2)$ & $58.9(14.9)$ & 0.74 & $65.3(14.6)$ & $53(15.2)$ & $\mathbf{0 . 0 4}^{*}$ \\
\hline STA & $5.8(5.6)$ & $8.3(6.5)$ & 0.23 & $7.9(6.4)$ & $5.5(3.9)$ & 0.24 \\
\hline GT & $27.6(15.8)$ & $29.5(15.1)$ & 0.91 & $42(12.9)$ & $22.8(12.6)$ & $\mathbf{0 . 0 0 3 ^ { * }}$ \\
\hline SS & $31.8(9.1)$ & $32.8(10.8)$ & 0.51 & $26.8(8.6)$ & $34.2(9.7)$ & $\mathbf{0 . 0 0 2}^{*}$ \\
\hline PT & $20.8(12.3)$ & $20.1(12)$ & 0.81 & $34.1(7.5)$ & $18.5(10.9)$ & $\mathbf{0 . 0 0 1}^{*}$ \\
\hline PI & $52.5(13.4)$ & $53.1(13.5)$ & 0.83 & $61.2(11.3)$ & $53(15.3)$ & $\mathbf{0 . 0 4 7}^{*}$ \\
\hline
\end{tabular}

OSF: One step forward, SVA: Spinal vertical axis, TK: Thoracic kyphosis, LL: Lumbar lordosis, SPA: Spinopelvic angle, STA: Spinal tilt angle, GT: Global tilt, SS: Sacral slope, PT: Pelvic tilt, PI: Pelvic incidence

Values are mean values (range), ${ }^{*}$ Statistically significant changes, SSA: Spinosacral angle

of patients and demonstrate the real extent of the balance problems compared to standard lateral X-rays.

\section{Ethics}

Ethics Committee Approval: Approved.

Informed Consent: Was taken.

Peer-review: Internally peer-reviewed.

\section{Authorship Contributions}

Surgical and Medical Practices: Ü.Ö.G., E.A., oncept: Ü.Ö.G., E.A., Design: Ü.Ö.G., E.A., Data Collection or Processing: Ü.Ö.G., Analysis or Interpretation: Ü.Ö.G., E.A., Literature Search: Ü.Ö.G., Writing: Ü.ö.G., E.A.

Conflict of Interest: No conflict of interest was declared by the authors.

Financial Disclosure: The authors declared that this study received no financial support.

\section{REFERENCES}

1. Bridwell KH, Baldus C, Berven S, Edwards C, Glassman S, Hamill C, et al. Changes in radiographic and clinical outcomes with primary treatment adult spinal deformity surgeries from two years to three- to five-years follow-up. Spine (Phila Pa 1976) 2010;35:1849-54.

2. Cho KJ, Suk SI, Park SR, Kim JH, Kang SB, Kim HS, et al. Risk factors of sagittal decompensation after long posterior instrumentation and fusion for degenerative lumbar scoliosis. Spine (Phila Pa 1976) 2010;35:1595-601.

3. Harding IJ. Understanding sagittal balance with a clinical perspective. Eur J Phys Rehabil Med 2009;45:571-82.

4. Horton WC, Brown CW, Bridwell KH, Glassman SD, Suk SI, Cha CW. Is there an optimal patient stance for obtaining a lateral 36;radiograp?A critical comparison of three techniques. Spine (Phila Pa 1976) 2005;30:427-33.
5. Husson JL, Mallet JF, Parent H, Cavagna R, Vital JM, Blamoutier A, et al. Applications in spinal imbalance. Orthop Traumatol Surg Res 2010.

6. Janssen MM, Drevelle X, Humbert L, Skalli W, Castelein RM. Differences in male and female spino-pelvic alignment in asymptomatic young adults: a three-dimensional analysis using upright low-dose digital biplanar X-rays. Spine (Phila Pa 1976) 2009;34:E826-32.

7. Korovessis P, Repantis T, Papazisis Z, lliopoulos P. Effect of sagittal spinal balance, levels of posterior instrumentation, and length of follow-up on low back pain in patients undergoing posterior decompression and instrumented fusion for degenerative lumbar spine disease:a multifactorial analysis. Spine (Phila Pa 1976) 2010;35:898-905.

8. Lafage V, Schwab F, Patel A, Hawkinson N, Farcy JP. Pelvic tilt and truncal inclination:two key radiographic parameters in the setting of adults with spinal deformity. Spine (Phila Pa 1976) 2009;34:599-606.

9. Lafage V, Schwab F, Skalli W, Hawkinson N, Gagey PM, Ondra S, et al. Standing balance and sagittal plane spinal deformity: analysis of spinopelvic and gravity line parameters. Spine (Phila Pa 1976) 2008;33:1572-8.

10. Lonner BS, Auerbach JD, Sponseller P, Rajadhyaksha AD, Newton PO.Variations in pelvic and other sagittal spinal parameters as a function of race in adolescent idiopathic scoliosis. Spine (Phila Pa 1976) 2010;35:E374-7.

11. Roussouly P, Nnadi C. Sagittal plane deformity: an overview of interpretation and management. Eur Spine J 2010;19:1824-36.

12. Schwab F, Lafage V, Patel A, Farcy JP. Sagittal plane considerations and the pelvis in the adult patient. Spine (Phila Pa 1976) 2009;34:1828-33.

13. Schwab F, Lafage V, Boyce R, Skalli W, Farcy JP. Gravity line analysis in adult volunteers: age-related correlation with spinal parameters, pelvic parameters, and foot position. Spine (Phila Pa 1976) 2006;31:E959-67.

14. Vialle R, Levassor N, Rillardon L, Templier A, Skalli W, Guigui P.Radiographic analysis of the sagittal alignment and balance of the spine in asymptomatic subjects. J Bone Joint Surg Am. 2005;87:260-7.

15. Videbaek TS, Bünger CE, Henriksen M, Neils $E$, Christensen FB. Sagittal Spinal Balance After Lumbar Spinal Fusion: The Impact of Anterior Column Support: Results From a Randomized Clinical Trial With an Eight-to Thirteen-Year Radiographic Fallow-up. Spine (Phila Pa 1976) 2011;36:183-91. 